\title{
On-Board Entry Trajectory Planning Expanded to Sub-Orbital Flight
}

\author{
Zuojun Shen* \\ Ping $\mathrm{Lu}^{\dagger}$ \\ Iowa State University \\ Ames, IA 50011-2271
}

\begin{abstract}
A methodology for on-board planning of sub-orbital entry trajectories is developed. The algorithm is able to generate in a time frame consistent with on-board environment a threedegree-of-freedom (3DOF) feasible entry trajectory, given the boundary conditions and vehicle modeling. This trajectory is then tracked by feedback guidance laws which issue guidance commands. The current trajectory planning algorithm complements the recently developed method for on-board 3DOF entry trajectory generation for orbital missions, and provides full-envelope autonomous adaptive entry guidance capability. The algorithm is validated and verified by extensive high fidelity simulations using a sub-orbital reusable launch vehicle model and difficult mission scenarios including failures and aborts.
\end{abstract}

\footnotetext{
${ }^{*}$ Postdoctoral Research Associate, Department of Aerospace Engineering. Email: zjshen@iastate.edu

${ }^{\dagger}$ Associate Professor, Department of Aerospace Engineering. Email: plu@iastate.edu. Associate Fellow AIAA
} 


\section{Introduction}

The traditional lifting entry guidance design, exemplified by the Space Shuttle entry guidance concept, ${ }^{1}$ relies on two main components. One is the planning of a reference drag profile on the ground before mission. The other is on-board closed-loop tracking of the reference drag profile. The off-line reference planning addresses the requirements of thermal considerations, loads and range. When the mission changes, the reference drag profile is re-generated in mission planning according to the new conditions. While highly successful in nominal operations, this approach would severely restrict the options that the vehicle has in aborts and other emergency situations because of the limitation of the pre-loaded reference profile. In recent years, a number of methods have been investigated that aim at on-board design of the reference for entry guidance. ${ }^{2-6}$ In the most recent related work by the authors, ${ }^{7}$ an algorithm is developed to generate on-board a three-degree-of-freedom (3DOF) constrained reference trajectory that satisfies all the trajectory constraints for entry flight and control authority limits. A centerpiece of that algorithm is the use of the so-called quasi-equilibrium glide condition (QEGC), a general phenomenon in lifting entry at high speeds. The range of flight conditions in which the QEGC is valid depends on the lifting capability of the vehicle. For vehicles with medium or higher lift-to-drag $(L / D)$ ratios, such as the Shuttle, the range could cover the entire entry flight. For vehicles with low $L / D$ ratios, however, the QEGC is usually valid above certain cut-off velocity (e.g., Mach 6-8). For this class of vehicles, this limitation could render the algorithm in Ref. 7 inapplicable to sub-orbital flight of these vehicles. While there is no magic separating line, the sub-orbital entry flight defined in this paper refers to an entry mission that begins below Mach 12. For such a mission the QEGC may not be applicable or may be valid only for too short a period, therefore a different approach is needed for in-flight entry trajectory design.

The need for planning sub-orbital entry trajectories can arise from flight testing of new entry vehicles, significantly off-nominal conditions, or aborts. During entry flight from orbit, unusually large environmental dispersions and vehicle modeling mismatches could cause large trajectory deviations from the nominal. In such a case it may be better off to redesign and fly a new reference trajectory starting from the current sub-orbital conditions. System failures during entry from orbit could-necessitate abort to an alternate landing site, thus require the vehicle to initiate a new sub-orbital trajectory different than the original reference trajectory. In launch abort scenarios, both downrange aborts and return-to-launchsite aborts consist of an entry phase that may start at a velocity significantly lower than the orbital velocity. In aborts it is also likely that the maneuver/control ability of the vehicle is impaired by the failures that caused the abort. The capability to autonomously re-design on-board a reference entry trajectory to adapt to the current conditions, alternate landing site if necessary, and the current maneuverability of the vehicle is crucial for the safe return of the crew and vehicle. The same desirable feature of the guidance system is also sought for the flight phase following entry flight, ${ }^{8}$ known as terminal area energy management (TAEM) phase.

This paper presents an approach for on-board planning of 3DOF reference entry trajectories for sub-orbital missions. The method in this paper complements the on-board entry trajectory design algorithm developed in Ref. 7 for entry at higher speeds, enabling the autonomous entry guidance system to have this key adaptive capability throughout the entry 
flight envelope. While this sub-orbital entry trajectory planning algorithm shares elements of heritage from the algorithm in Ref. 7, major differences are inevitable because the QEGC is no longer used here. Some of the primary driving factors in design of sub-orbital entry trajectories also differ from those for orbital entry flight. Since sub-orbital flight is much shorter, and thermal consideration is no longer a key concern, the main criterion for the reference trajectory is feasibility, meaning that it must be intrinsically flyable, and satisfy all the boundary conditions imposed. The key ingredients in the algorithm proposed include dividing the trajectory into a constant bank angle phase and analytical representation phase, and an optional final compensation update that further reduces terminal condition errors. In the analytical representation phase the altitude-vs-velocity profile is represented by a polynomial. This polynomial is carefully constructed so that its flyability is maximized. Two sequential one-parameter searches for the bank angle magnitude of the constant bank phase and for the point where the bank angle changes sign (referred to as bank reversal) are the main iterations. Extensive tests using high fidelity simulations with the model of the $\mathrm{X}-33$, a sub-orbital reusable launch vehicle, have been conducted to verify the algorithm. The algorithm converges in a fraction of one second on a desktop computer. Some of the test scenarios presented in this paper include 6DOF Monte Carlo simulations of trajectories under all commonly examined dispersions, and scenarios involving engine-out aborts and control surface actuator failure. The entry guidance system with the on-line trajectory planning capability succeeds in all these tests.

\section{Problem Formulation}

\section{A. Entry Dynamics}

The well-known 3DOF point-mass dynamics of the entry vehicle over a spherical Earth are described by the following dimensionless equations of motion ${ }^{9}$

$$
\begin{aligned}
\dot{r} & =V \sin \gamma \\
\dot{\theta} & =\frac{V \cos \gamma \sin \psi}{r \cos \phi} \\
\dot{\phi} & =\frac{V \cos \gamma \cos \psi}{r} \\
\dot{V} & =-D-\frac{\sin \gamma}{r^{2}} \\
\dot{\gamma} & =\frac{1}{V}\left[L \cos \sigma+\left(V^{2}-\frac{1}{r}\right)\left(\frac{\cos \gamma}{r}\right)\right] \\
\dot{\psi} & =\frac{1}{V}\left[\frac{L \sin \sigma}{\cos \gamma}+\frac{V^{2}}{r} \cos \gamma \sin \psi \tan \phi\right]
\end{aligned}
$$

where $r$ is the radial distance from the center of the Earth to the vehicle, normalized by the radius of the Earth $R_{0}=6378(\mathrm{~km})$. The longitude and latitude are $\theta$ and $\phi$, respectively. The Earth-relative velocity $V$ is normalized by $V_{c}=\sqrt{g_{0} R_{0}}$ with $g_{0}=9.81$ $\mathrm{m} / \mathrm{sec}^{2}$. The terms $D$ and $L$ are the aerodynamic drag and lift accelerations in $g$ 's, i. e., $D=\rho\left(V_{c} V\right)^{2} S_{\text {ref }} C_{D} /\left(2 m g_{0}\right)$ and $L=\rho\left(V_{c} V\right)^{2} S_{r e f} C_{L} /\left(2 m g_{0}\right)$, where $\rho$ is the atmospheric density, $S_{\text {ref }}$ the reference area of the vehicle, and $m$ the mass of the RLV. Note that $D$ and 
$L$ are also functions of $\alpha$, the angle of attack, through the dependence of the drag and lift coefficients $C_{D}$ and $C_{L}$ on $\alpha$. The flight path angle is $\gamma$ and $\sigma$ the bank angle. The velocity azimuth angle $\psi$ is measured from the North in a clockwise direction. The differentiation is with respect to the dimensionless time $\tau=t / \sqrt{R_{0} / g_{0}}$. The Earth rotation terms have been ignored in above equations because their effects on sub-orbital flight are negligible.

\section{B. Trajectory Constraints}

The reference entry trajectory should start at the current conditions, and terminate at a specified distance from the landing site where the guidance is handed over to the TAEM guidance system. At the TAEM interface, the entry trajectory must have correct conditions to ensure that successful TAEM and approach/landing flight is possible. These conditions form the terminal conditions for the entry trajectory as

$$
\begin{aligned}
r_{f} & =r_{T A E M} \\
V_{f} & =V_{T A E M} \\
s_{f} & =s_{T A E M}
\end{aligned}
$$

where $s_{f}$ is the final value of range-to-go $s_{\text {togo }}$, defined to be the range from the vehicle position to the tangency of the heading alignment cone (HAC) near an end of the runway. The coordinates of the center of the HAC are known. The TAEM altitude $r_{T A E M}$, velocity $V_{T A E M}$ and range-to-HAC $s_{T A E M}$ are all specified for a given vehicle. In addition, the Earth-relative velocity vector at the TAEM interface should be pointing nearly to the HAC tangency. Let $\Delta \psi_{f}$ be the difference between the velocity azimuth angle and the line-of-sight angle from the vehicle to the HAC at the TAEM interface. This condition is then represented by

$$
\left|\Delta \psi_{f}\right| \leq \Delta \psi_{T A E M}
$$

This condition stipulates that the final velocity vector should be directed at the HAC within a given tolerance $\triangle \psi_{T A E M}$.

The bank angle magnitude at the TAEM interface often times is also another parameter required not to be excessively large. Too large a $\sigma_{f}$ could result in large transient response for the TAEM guidance and control. Thus, the constraint

$$
\left|\sigma_{f}\right| \leq \sigma_{\text {TAEM }}
$$

for a given $\sigma_{T A E M}>0$ may also be imposed.

Inequality trajectory (path) constraints may also need to be considered. For sub-orbital flight, possible path constraints include

$$
\begin{gathered}
|L \cos \alpha+D \sin \alpha| \leq n_{z_{\max }} \\
q \leq q_{\max }
\end{gathered}
$$

The constraint Eq. (12) is on the aerodynamic load (in $g$ 's) in the body-normal direction. Depending on the vehicle configuration and mission, this constraint may be replaced by the total load constraint

$$
\sqrt{L^{2}+D^{2}} \leq n_{\max }
$$


Constraint Eq. (13) is on the dynamic pressure with $q=\rho\left(V_{c} V\right)^{2} / 2$. The parameters $n_{z_{\max }}\left(n_{\max }\right)$ and $q_{\max }$ are vehicle-dependent constants. Note that the heat constraint which is a primary concern in orbital entry flight is not listed here, because in sub-orbital flight it is usually not active.

\section{Trajectory Planning Problem}

The sub-orbital entry trajectory planning problem is defined as follows: given the initial conditions, vehicle modeling data, terminal conditions at the TAEM interface, find the state history of $\mathbf{x}=\{r \theta \phi V \gamma \psi\}^{T}$ and the corresponding trajectory control $\mathbf{u}= \begin{cases}\sigma & \alpha\}^{T} \text { such }\end{cases}$ that:

1. The state and control profiles $\mathbf{x}(t)$ and $\mathbf{u}(t)$ satisfy the 3DOF equations of motion Eqs. (1-6);

2. The pair $[\mathbf{x}(t) \mathbf{u}(t)], t_{0} \leq t \leq t_{f}$, satisfies the current flight conditions at $t_{0}$, and all the required TAEM interface conditions at $t_{f}$ as specified in the preceding section;

3. Both $\sigma$ and $\alpha$ profiles do not exceed the flight control system authority in terms of the maximum magnitudes, rates and accelerations of $\sigma$ and $\alpha$.

A pair $[\mathbf{x}(t) \mathbf{u}(t)]$ that meets the above conditions is called a feasible trajectory. For on-board applications, the algorithm must find a feasible trajectory quickly and reliably. Two implicit assumptions are that (1) for the given conditions and vehicle model, a feasible trajectory exists; (2) the entry flight is lifting flight, i.e., $L / D \neq 0$. The third assumption to be made is that a nominal $\alpha$-versus-velocity (or Mach number) profile is available, and limited variations about this nominal profile are allowable. This assumption is not a necessity for the algorithm, but for practical purpose. An arbitrarily designed $\alpha(t)$ profile may not be compatible with the requirements of flight trim conditions. And a nominal $\alpha$ profile, once determined for a given vehicle, typically does not change significantly from mission to mission.

\section{Algorithm Development}

\section{A. Overview}

The algorithm generates a sub-orbital entry trajectory in 2 major steps plus an optional final step:

1. Design the longitudinal profiles for velocity and altitude that meet the range requirement and TAEM conditions Eqs. (7-9), and find the associated bank angle magnitude and angle of attack profile.

2. Search for the appropriate bank reversal point based on the heading offset with respect to the HAC condition (10) to determine the sign of the bank angle $\sigma$. Complete the 3DOF trajectory by integrating Eqs. (1-6) with $\sigma$ whose magnitude is that required to track the longitudinal profiles obtained in Step 1.

3. When necessary, further improve the accuracy of the TAEM range condition (9) by slightly adjusting the angle of attack profile if the TAEM range error exceeds tolerance. 
Step 1 is further divided into two phases: a constant-bank phase and an analytical representation phase. The constant-bank phase is a period where a to-be-determined constant bank angle is used. This phase serves as a transition period from the given initial conditions to the analytical representation phase, where the desired altitude-vs-velocity profile of the trajectory is approximated by a polynomial. This polynomial is designed to meet the TAEM conditions (7) and (8) and account for the path constraints when necessary. The flyability of this analytical representation will be analyzed. The value of the constant bank angle is searched so that the combination of these two phases gives the correct TAEM conditions as in Eqs. (7-9).

Step 2 is much like the corresponding part of the algorithm in Ref. 7 . In this step, another one-parameter search is iteratively performed to determined the bank reversal point while tracking the altitude-vs-range and velocity-vs-range profiles by feedback control laws in $\sigma$ and $\alpha$. When the correct bank reversal point is found, the integrated trajectory will have also a TAEM heading offset satisfying (10), and this will be the final generated reference trajectory (provided Step 3 is not opted).

Step 3 is optional. In some cases the error in the range condition (9) from Step 2 at the specified TAEM energy could be larger than tolerance. This error is chiefly caused by less accurate tracking of the longitudinal profiles due to the bank reversal taking place too close to the TAEM interface. In those cases the error in the range is used to adjust the angle of attack profile to reduce the TAEM range error.

The details in each step, Steps 1 and 3 in particular, are given below.

\section{B. Longitudinal Profiles}

The longitudinal trajectory profiles are those for velocity, altitude, and the corresponding bank angle magnitude and $\alpha$ histories. The flight path angle profile will then be determined from $r$ and $V$ through Eq. (1). These profiles should satisfy dynamic Eqs. (1), (4) and (5). Note that the sign of $\sigma$ cannot be determined by the longitudinal profiles, but is left for Step 2 of the algorithm. With the assumption of small heading offset with respect to $\mathrm{HAC}, \Delta \psi$, the range-to-HAC history is also defined from the longitudinal profile. Figure 1 shows in the velocity-altitude space a typical sub-orbital entry trajectory, the boundaries of the path constraints (12-13), and the formation of the constant-bank and analytical representation phases.

\section{B1. Constant-Bank Phase}

In this phase a constant bank angle $\sigma_{0}$ and the nominal $\alpha$ profile are used to numerically integrate the $3 \mathrm{DOF}$ equations of motion (1)-(6) with the given entry conditions. The sign of $\sigma_{0}$ is so chosen that the aerodynamic lift contributes to reduce the magnitude of $\Delta \psi$, the heading offset to the HAC. Starting from a first-cut guess of $\sigma_{0}$ (such as $\left|\sigma_{0}\right|=40 \mathrm{deg}$ ), the integration is continued until at a point where the following condition is satisfied

$$
\left|\frac{d r}{d V}-\frac{r-r_{T A E M}}{V-V_{T A E M}}\right|<\varepsilon
$$

where $\varepsilon>0$ is a small preselected value, and from Eqs. (1) and (4) $d r / d V$ is calculated by

$$
\frac{d r}{d V}=\frac{V \sin \gamma}{-D-\sin \gamma / r^{2}}
$$


The condition (15) is continuously checked during the integration. The point where (15) is met is called the transition point which marks the end of the constant-bank angle phase and the beginning of the analytical representation phase. The geometric interpretation of condition (15) is that at the transition point, the trajectory is pointing at the TAEM point in the velocity-altitude space (cf. Fig. 1). Another benefit of such a choice is to aid the flyability analysis in Section B3.

As will be seen in the next section, the trajectory conditions at the transition point help uniquely define a polynomial in $r$ and $V$ in the next phase. The computation in the next phase will also provide clear information as to how $\sigma_{0}$ should be adjusted in order to meet the TAEM constraints Eqs. (7-9). The above process is then repeated with the updated $\sigma_{0}$. When the process is completed, the integrated longitudinal profiles, $\sigma_{0}$, and $\alpha$ from the entry point to the transition point, as well as the range-to-HAC history $s_{\text {togo }}$, are all obtained and stored for later use.

\section{B2. Analytical Representation Phase}

In this phase a 4 th order polynomial is used to represent the desired altitude-vs-velocity profile connecting the transition point to the TAEM point

$$
r=a_{4} V^{4}+a_{3} V^{3}+a_{2} V^{2}+a_{1} V+a_{0}
$$

Five conditions are needed to determine the coefficients $a_{i}$. Denote the known altitude and velocity at the transition point $T$ by $r_{T}$ and $V_{T}$. Let the midway point in velocity between $V_{T}$ and the specified TAEM velocity $V_{T A E M}$ be $V_{c}$, i.e.,

$$
V_{c}=0.5\left(V_{T A E M}+V_{T}\right)
$$

Suppose that at $V_{c}$, the boundary of the dynamic pressure constraint (13) dominates that of the load constraint (12) as depicted in Fig. 1. Choose the altitude $r_{c}$ at $V_{c}$ such that the dynamic pressure at the midway point $C$ is the average value of the dynamic pressure at point $T$ and the TAEM point. The choice of $r_{c}$ will be based on average value of load if the load constraint dominates the dynamic pressure constraint at $V_{c}$. Such a choice of of $r_{c}$ ensures that the midpoint of the curve (17) in the $V-r$ space will lie above the constraint boundary, therefore the path constraint is not violated at this point.

Three conditions are already given or known that will be used to determined the coefficients in Eq. (17): $\left(r_{T A E M}, V_{T A E M}\right),\left(r_{T}, V_{T}\right)$, and $(d r / d V)_{T}$ (the value of $d r / d V$ at point $T$ ). The remaining two conditions will be from the values of $d r / d V$ at the TAEM point, denoted by $f_{0}$, and $d^{2} r / d V^{2}$ at the TAEM point, denoted by $g_{0}$. It is not difficult to show that from the expressions of $f_{0}$ and $g_{0}$ by differentiating (17), the altitude at $V_{c}$ can be expressed as

$$
r_{c}=\frac{5}{16} y_{1}-\frac{1}{16} x_{1} f_{1}+\frac{1}{4} f_{0} x_{1}+\frac{1}{32} g_{0} x_{1}^{2}+r_{T A E M}
$$

where $x_{1}=V_{T}-V_{T A E M}, y_{1}=r_{T}-r_{T A E M}$, and $f_{1}=(d r / d V)_{T}$. The value of $r_{c}$ is already determined as described above, as well as other quantities except for $f_{0}$ and $g_{0}$. On the other hand, the vehicle dynamics dictate that $d r / d V$ is given by Eq. (16) and

$$
\begin{aligned}
\frac{d^{2} r}{d V^{2}} & =\frac{1}{-\dot{V}}\left(-\sin \gamma-\frac{1}{\dot{V}} \dot{\gamma} V \cos \gamma\right) \\
& +\frac{V \sin \gamma}{\dot{V}^{2}}+\frac{\dot{\gamma} \cos \gamma}{\dot{V} r^{2}}+\frac{2 \dot{r} \sin \gamma}{\dot{V} r^{3}}+D_{V}
\end{aligned}
$$


where $D_{V}=\partial D / \partial V$. From Eq. (16) it is clear that $f_{0}=(d r / d V)_{T A E M}$ is defined if $\gamma_{T A E M}$ is selected, because $r_{T A E M}$ and $V_{T A E M}$ are given. When replacing $\dot{r}, \dot{V}$ and $\dot{\gamma}$ in Eq. (19) by Eqs. (1), (4) and (5), we see for the same reason that $g_{0}=\left(\dot{d}^{2} r / d V^{2}\right)_{T A E M}$ is dependent only on $\gamma_{T A E M}$ and $\sigma_{T A E M}$. We will set $\sigma_{T A E M}$ to be a relatively small value or zero to reflect the desire of Eq. (11). Therefore both $f_{0}$ and $g_{0}$ are functions of $\gamma_{T A E M}$, and condition (18) constitutes an equation for $\gamma_{T A E M}$. Solving (18) numerically gives $\gamma_{T A E M}$, and $f_{0}$ and $g_{0}$ in turn. It should be noted that the solution process converges quickly despite the transcendental nature of Eq. (18). This is due to the fact that the term $g_{0} x_{1}^{2} / 32$ is smaller compared to other terms because $0<x_{1}<1$ in dimensionless variables, thus close initial guess of the solution can be obtained by ignoring the term $g_{0} x_{1}^{2} / 32$ first.

Now that the desired $r$-vs- $V$ profile (17) is determined, we turn our attention to the range requirement. The range-to-go to the HAC, $s_{\text {togo }}$, is defined to be the arc length in radian along a great circle from the current position of the vehicle to the HAC. The differential equation for $s_{\text {togo }}$ is

$$
\dot{s}_{\text {togo }}=-\frac{V \cos \gamma \cos \Delta \psi}{r}
$$

where $\Delta \psi$ is the heading offset angle with respect to the HAC. The usual approximation of $\cos \Delta \psi \approx 1$ proves to be still valid in sub-orbital flight. Hence combining Eq. (4) and the above equation gives

$$
\frac{d V}{d s_{\text {togo }}}=\frac{r}{V \cos \gamma}\left(D+\frac{\sin \gamma}{r^{2}}\right)
$$

Note that the independent variable $s_{\text {togo }}$ is decreasing. The drag $D$ is a dominating factor in above equation since $r \approx 1$ in dimensionless form. Also $\gamma$ is no longer required to be small as usually assumed in orbital entry, a condition for the QEGC to be valid. At any given $V, r$ is computed from Eq. (17). The drag $D$ then is calculated with $r, V$, and the nominal $\alpha$ profile. Finally $\gamma$ is evaluated by using Eq. (16) with $d r / d V$ obtained from differentiating Eq. (17) once with respect to $V$. In other word, the right hand side of the differential equation (21) is completely defined at any given $V$.

The value of $s_{\text {togo }}$ at point $T$, the beginning of this phase is already known from the constant-bank phase. The final value of $s_{\text {togo }}$ is specified by Eq. (9). The Eq. (21) can now be numerically integrated in this interval and evaluate the end velocity. If the end velocity is higher than the required value $V_{T A E M}$, it suggests that a larger value of $\left|\sigma_{0}\right|$ in constant-bank phase should be used. This is because a larger $\left|\sigma_{0}\right|$ in that phase will effectively lower the altitude $r_{T}$ at the transition point. A lower $r_{T}$ will then cause the curve (17) to be lower in the velocity-altitude space. The lowering of the the $r$-vs- $V$ profile in turn increases the drag $D$. The net effect is that the velocity will decrease faster. The reverse is also true. The iteration involves both constant-bank phase and analytical representation phase. A bisection algorithm will converge quickly in finding the correct $\sigma_{0}$ because of this monotonic functional relationship.

Once the iteration stops, the TAEM range condition (9) and velocity condition (7) are satisfied. The condition on altitude (7) is also implicitly met because $r$ is calculated from Eq. (17) and condition (7) is used in constructing the curve (17). In addition to the obtained $r\left(s_{\text {togo }}\right)$ and $V\left(s_{\text {togo }}\right)$ profiles, the corresponding $\gamma\left(s_{\text {togo }}\right)$ profile is again computed from Eq. (16) with $d r / d V$ obtained by differentiating (17) once with respect to $V$. One more differentiation of (17) with respect to $V$ gives $d^{2} r / d V^{2}$, which together with Eq. (19) can be used 
to back out the bank angle magnitude profile $\left|\sigma\left(s_{\text {togo }}\right)\right|$.

\section{B3. Flyability of Analytical Altitude-Velocity Profile}

The analytical profile (17) is not yet a part of the final trajectory. The final trajectory for this phase is the result of numerical integration of the dynamic equations (1-6) with $\sigma$ required to track the profile (17), as will be outlined in the next section. But for (17) to be a close approximation to the actual trajectory, the curve (17) must be flyable, at least for the most part. Flyability here means that when the altitude and velocity of the vehicle are on the curve, the required trajectory control for the vehicle to stay on the curve is within the capability of the vehicle.

For entry flight, the chief trajectory control comes from the modulation of the bank angle. In the $r-V$ space, the effect of the bank angle appears in $d^{2} r / d V^{2}$ as in Eq. (19) through the presence of $\dot{\gamma}$. For fixed values of $r, V, \gamma$, and $\alpha$, the range of variations of $d^{2} r / d V^{2}$ when $\sigma$ varies defines the flyability in longitudinal direction. We will consider the range of $\sigma$ between $0 \mathrm{deg}$ and $90 \mathrm{deg}$ for demonstration (it is possible to have $\sigma>90 \mathrm{deg}$ ). With the nominal $\alpha$ profile and for every point on the curve (17), the range of possible values of $d^{2} r / d V^{2}$ is limited by value of $d^{2} r / d V^{2}$ corresponding to $\sigma=0 \mathrm{deg}$, and the value to $\sigma=90$ deg. Figure 2 shows in dashed lines the upper and lower boundaries of $d^{2} r / d V^{2}$ with respect to $V$ when $r$ is from (17).

The physical interpretation of these boundaries is that they represent the limits of acceleration for the vehicle to pull up and dive, respectively. The solid line is the actual value of $d^{2} r / d V^{2}$ along the curve (17) for each $V$. When the solid line is inside the boundaries as shown in Fig. 2, it indicates that the vehicle has the required trajectory control capability to fly the curve (17). In Fig. 2 the solid line meets the upper boundary at the TAEM point because we set the $\sigma_{T A E M}=0$ in constructing the curve (17).

The question to be asked is how the flyability of the profile (17) can be improved if necessary. This is done by moving the two control points $T$ and $C$ inside the flyability corridor if needed. Note that the TAEM point is always inside the corridor by the way the value of $g_{0}=\left(d^{2} r / d V^{2}\right)_{T A E M}$ is chosen in the design of the profile. Recall that the point $T$ is determined from the constant-bank phase, and the values of $r_{c}$ and $V_{c}$ at point $C$ are determined as $T$ is fixed. Recall also that the transition point $T$ is selected by the condition $f_{T}=y_{1} / x_{1}$ (cf. Eq. (15). With this, it can be shown that the values of $d^{2} r / d V^{2}$ at point $T$ and $C$ on the profile (17) are related by

$$
\left.\frac{d^{2} r}{d V^{2}}\right|_{T}=\left[g_{0}-\frac{6}{x_{1}}\left(\frac{y_{1}}{x_{1}}-f_{0}\right)\right]=-\left.2 \frac{d^{2} r}{d V^{2}}\right|_{C}
$$

Between the two free parameters $f_{0}$ and $g_{0}, f_{0}$ (equivalently, $\gamma_{T A E M}$ ) can be chosen to meet the condition (18). The above proportionality condition indicates that $g_{0}$ (alternatively, $\left.\sigma_{T A E M}\right)$ may be selected to move simultaneously the the values of $d^{2} r / d V^{2}$ at the two points $T$ and $C$ into the flyability corridor if necessary. Once all the three points $T, C$ and TAEM are all inside the flyability corridor as in Fig. 2, it is likely that the entire curve representing the value of $d^{2} r / d V^{2}$ of the profile (17) will remain inside the corridor, given the relatively short range of $V$ in this phase in sub-orbital flight.

\section{Completion of the 3DOF Trajectory}


At this time the longitudinal profiles in the constant-bank phase and the desired longitudinal profiles based on (17) in the analytical representation phase are available. The lateral profiles and when to change the sign $\sigma$ remain to be determined so that the TAEM condition (10) is also met. The strategy to achieve these objectives is essentially the same as the counterpart in the algorithm in Ref. 7 . We will only outline the approach here. The reader is referred to Ref. 7 for detail.

Starting from the entry point $E$, linear, time-varying feedback control laws for $|\sigma|$ and $\alpha$ are used to track the profiles $r\left(s_{\text {togo }}\right)$ and $V\left(s_{\text {togo }}\right)$ obtained in Sections B1 and B2. Initially the sign of $\sigma$ is the same as that of $\sigma_{0}$ used in the constant-bank phase. The computed $\sigma$ and $\alpha$ are unutilized to numerically integrate the dynamic equations (1-6). The the value of range $s_{\text {togo }}$ at the point where the bank reversal takes place is found in a one-parameter search. At this point the sign of $\sigma$ is changed at the maximum allowable rate to the opposite. The point of bank reversal is found so that the heading alignment condition (10) is met at the TAEM interface.

At the conclusion of this step, if the TAEM range meets the condition (9) within the given tolerance at the TAEM energy specified by conditions (7) and (8), a complete 3DOF entry trajectory has been found. Because the trajectory satisfies the equations of motion, it will be intrinsically flyable.

\section{E. TAEM Condition Precision Refinement}

In some minority cases when the process in Section D is completed, the TAEM range will have larger than acceptable errors. These errors are most likely to be caused by the fact that the bank reversal point is too close to the TAEM interface. During the bank reversal, the chief trajectory control $\sigma$ is rate-saturated. Consequently $\sigma$ renders no effective trajectory control during this period which can be considerable compared to the total entry flight time (see the example of mich10d1 mission in the next section). When bank reversal occurs too close to the TAEM interface, the feedback control laws used to track the longitudinal profiles in Section D simply do not have sufficient time to remove the tracking errors accumulated during the bank reversal before the entry flight is terminated. In such a case it is usually futile to attempt to move the bank reversal point away from TAEM, because the entry conditions and vehicle lifting capability have largely dictated where the bank reversal has to be.

Suppose that at the specified TAEM energy, there exists an unacceptable range error

$$
\Delta s_{f}=s_{f}-s_{T A E M}
$$

Since this error is inherent to the problem as indicated above, it would be more effective if an appropriate bias is built into specifying the TAEM range requirement in trajectory planning such that, after accounting for the effects of the bank reversal, the actual range at the TAEM is $s_{T A E M}$ as originally stipulated. The information on the case-dependent bias is already contained in $\Delta s_{f}$ above. Thus a modified TAEM range requirement is given by

$$
s_{T A E M}^{\prime}=s_{T A E M}-\Delta s_{f}
$$

The longitudinal profiles found in Sections B1 and B2 with the nominal $\alpha$ profile will no longer meet the new TAEM range condition. The strategy of adapting to the updated 
$s_{T A E M}^{\prime}$ is to keep the constant-bank phase unchanged, and only modify slightly the $\alpha$ profile in the analytical representation phase by a constant $\delta \alpha$. Define the specific energy to be $e=1 / r-V^{2} / 2$. It can be readily shown that

$$
\frac{d s_{\text {togo }}}{d e}=-\frac{\cos \gamma}{r D}
$$

when $\cos \Delta \psi \approx 1$ is used. Along the already found profile (17), increasing $\alpha(\delta \alpha>0)$ elevates the drag $D$, thus reduces the range, and vice versa. Hence an appropriate $\delta \alpha$ can be quickly determined using the bi-section method to accommodate the new range condition $s_{\text {TAEM }}^{\prime}$. The longitudinal profiles $r\left(s_{\text {togo }}\right), V\left(s_{\text {togo }}\right)$ and $\gamma\left(s_{\text {togo }}\right)$ plus $\left|\sigma\left(s_{\text {togo }}\right)\right|$ and $\alpha\left(s_{\text {togo }}\right)$ in the analytical representation phase are then updated accordingly.

After the above updates, the steps in Section $\mathrm{D}$ are repeated to complete the 3DOF trajectory. These minor updates will not alter the point of the bank reversal by appreciable extent, thus the range error characteristics remain the same. Since a corresponding range bias has been built into the longitudinal profile planning, the actual TAEM range will generally be improved to be closer to the specified value $s_{T A E M}$.

As a summary, Fig. 3 provides a flowchart that captures the main steps in the algorithm.

\section{Algorithm Tested}

The test cases presented in this paper use the model and mission profiles of the X33, a half-scale prototype of single-stage reusable launch vehicle (RLV) with lifting-body configuration. The X-33 was to be launched from the Edwards Air Force Base (AFB), accelerated to about Mach 9, and then glide to land horizontally at the Michael Army Air Field (AAF) in Utah. The trajectory sequence consists of ascent, transition, entry, TAEM, and approach/landing. High fidelity simulation software for the X-33, called Marshall Aerospace VEhicle Representation In C (MAVERIC), was developed at NASA Marshall Space Flight Center. Even though the X-33 program was eventually canceled, MAVERIC is continued to be used as a test bed for advanced RLV guidance and control technology development. In particular, for sub-orbital entry guidance technology assessment, a suite of 19 tests were compiled that were based on the X-33 missions. ${ }^{10}$ These tests include 6 DOF nominal mission dispersion studies, large thrust dispersions, engine failures, control actuator failures, and significant aerodynamic modeling mismatches. The performance of the entry guidance algorithm is judged by the TAEM condition precision, observance of path constraints, thermalstructural indicators, control surface activities, and reaction control system propellant usage. Most of the tests are stressful and some are impossible to handle by a conventional entry guidance algorithm without on-board trajectory planning capability. In fact, the baseline entry guidance algorithm for the X-33 only managed to get $8.4 \%$ of the total scores, failing most of the tests where it was evaluated. In contrast, the entry guidance algorithm incorporating the trajectory planning algorithm proposed in this paper passed all the tests, and achieved overall $91 \%$ of the total scores. Some of the test scenarios and results are presented in the following to demonstrate the performance of the algorithm.

\section{A. X-33 Nominal Missions}


Two mission profiles of the X-33 are considered. The baseline X-33 mission is called mich10a1. After the cut-off of the engines at about Mach 9, the entry flight starts following a 25-second transition period. The guidance during the transition period is open-loop. The entry flight lasts about 4 minutes. The other mission, mich10d1, is designed to delay the transition from laminar to turbulent flow, a part of an experiment to assess the properties of the thermal protection system. The open-loop transition period of mich10d1 mission is thus about 90 seconds long, and the closed-loop entry flight lasts for only 2 minutes. The nominal angle of attack profiles are different for the two missions. The nominal landing site for both missions is Michael AAF.

The TAEM conditions, path constraints, and control authority constraints are as follows:

- TAEM altitude of $29.344 \mathrm{~km}$ for mich10a1 and $29.438 \mathrm{~km}$ for mich10d1;

- TAEM range-to-HAC of $55.56 \mathrm{~km}(30 \mathrm{~nm})$;

- TAEM velocity of $926 \mathrm{~m} / \mathrm{s}$ for mich10a1 and $924.6 \mathrm{~m} / \mathrm{s}$ for mich10d1;

- TAEM heading angle offset with respect to the HAC desired to be less than $5 \mathrm{deg}$, but no greater than $10 \mathrm{deg}$ in any case;

- peak normal acceleration not to exceed $2.5 \mathrm{~g}$;

- peak dynamic pressure not to exceed $14,364 \mathrm{~N} / \mathrm{m}^{2}$ (300 psf);

- TAEM bank angle magnitude not to exceed $50 \mathrm{deg}$;

- maximum rates of $5 \mathrm{deg} / \mathrm{s}$ for bank angle and angle of attack;

- maximum acceleration of $3.5 \mathrm{deg} / \mathrm{s}^{2}$ and $2.0 \mathrm{deg} / \mathrm{s}^{2}$ for bank angle and angle of attack, respectively.

The entry trajectory planning algorithm discussed in the preceding sections was implemented as a part of the entry guidance module in MAVERIC. At the beginning of the entry flight, a $3 \mathrm{DOF}$ reference trajectory was generated on-line based on the current navigation data. This trajectory was subsequently tracked by the feedback guidance laws described in Ref. 11. The guidance laws provided bank angle and angle of attack commands throughout the entry flight.

The 6DOF simulation results using MAVERIC are shown in Figs. 4-7 for the two missions. The X-33 baseline flight control system design was used in the 6DOF simulations. Average winds at Edwards AFB and mean winds from the Global Reference Atmospheric Model $^{12}$ (GRAM) were included in the simulations. Also plotted in these figures in dashed lines are the reference profiles generated by the trajectory planning algorithm, and, the guidance commands generated by the guidance laws in dotted lines in Figs. 5 and 7 . The control system tracks the guidance commands which are not exactly the same as the reference values because of trajectory dispersions. As evident from the figures, the 6DOF trajectories and the 3DOF reference trajectories match quite well, given the nontrivial differences between the $3 \mathrm{DOF}$ and $6 \mathrm{DOF}$ dynamics plus the control system dynamics. 
Some of the TAEM condition errors for both the reference trajectories and the $6 \mathrm{DOF}$ simulated trajectories are listed in Table 1. The TAEM interface is reached when the Earthrelative velocity (without counting for wind velocity) is equal to the specified TAEM velocity, therefore no TAEM velocity errors are shown in Table 1 . The precision levels seen in the Table are excellent in the context of $6 \mathrm{DOF}$ entry flight. As can be observed from Figs. 5 and 7, the magnitudes of the TAEM bank angles in both cases are well within the 50-deg desirable range. Although not plotted in the figures or listed in the Table, the peak normal load and peak dynamic pressure along the 6DOF trajectories are all within their respective limits. The computation times required to generate the two reference trajectories on a $800 \mathrm{MHz}$ desktop computer are also in the Table. The algorithm implementation was not optimized or streamlined for efficiency. Yet in both cases the computation time is less than 0.5 second.

Table 1: TAEM condition precision and CPU time

\begin{tabular}{lcccc}
\hline Case & $\Delta \psi_{f}(\mathrm{deg})$ & $\Delta s_{f}(\mathrm{~km})$ & $\Delta r_{f}(\mathrm{~km})$ & CPU time (sec) \\
\hline \hline mich10a1-Ref. & -0.49 & 0.616 & 0.118 & 0.328 \\
mich10a1-6DOF & 0.74 & 1.848 & -0.07 & \\
mich10d1-Ref. & 0.07 & 0.694 & 0.664 & 0.484 \\
mich10d1-6DOF & 0.63 & -0.43 & -0.76 & \\
\hline \hline
\end{tabular}

Monte Carlo simulations evaluate how the guidance system respond to uncertainty and dispersions. Random perturbations in propulsion, jet effects, aerodynamic coefficients, navigation data, mass properties, winds and atmospheric properties are included in the simulations. These dispersions cause the ascent trajectory to be different in each case, resulting in different entry conditions. The variations in the entry conditions can be quite appreciable, as documented by Dukeman. ${ }^{13}$ The same uncertainties (except for those in propulsion) affect the entry flight as well. For each case, the entry trajectory planning algorithm generates online a 3DOF reference trajectory based on the actual entry conditions (hence the reference trajectory is different each time). The guidance laws track the reference after that. The TAEM conditions of $100^{-}$dispersed $6 \mathrm{DOF}$ trajectories for mich10a1 and 10d1 mission each are given in Figs. 8 and 9. In Fig. 8 the values of TAEM altitude versus range-to-HAC are plotted. Any TAEM range within $5.5 \mathrm{~km}(3 \mathrm{~nm})$ of the specified value and any TAEM altitude within $0.914 \mathrm{~km}$ (3000 ft) of the targeted TAEM altitude are considered equally good. Any TAEM range-to-HAC outside $13 \mathrm{~km}(7 \mathrm{~nm})$ and altitude outside $2.134 \mathrm{~km}(7000$ $\mathrm{ft}$ ) of the respective specified values (in plus and minus directions) are regarded as failing the criterion. By these standards it can be seen that all of the dispersed cases of the 200 trajectories pass the test, and most of them are on the target. Figure 9 shows the TAEM heading angle errors with respect the the HAC versus the the actual heading angle for the 200 trajectories. The figure reveals that while the trajectories for mich10a1 mission approach the HAC from different directions than those of the trajectories for mich10d1 mission, as indicated by the different final heading angles, the heading-to-HAC errors are all within \pm 10 deg range, with mich10d1 trajectories actually fairing better in this criterion. Other criteria are all well met by the majority of the trajectories. In fact, the two sets of dispersion tests score $97.2 \%$ and $95.4 \%$ out of $100 \%$ respectively when all the scoring criteria are measured. 


\section{B. Cases Simulating System Failures}

Fifteen of the ninteen test scenarios based on X-33 sub-orbital missions are for various cases of large thrust dispersions, engine failures, actuator failures, and substantial mismodeling of aerodynamic coefficients. ${ }^{10}$ The goal is to evaluate the ability of the entry guidance algorithms to save the vehicle in these stressful situations. In a sense, these are the situations where a fully autonomous entry guidance system with on-board adaptive trajectory planning capability can set apart from the conventional entry guidance system.

Two of the cases to be shown here involve engine failure. The X-33 has two linear aerospike engines. Should one fail (power pump out, PPO for short), the propellant can be pumped to the other engine to continue the ascent with longer ascent flight time until the propellant is burnt out. MAVERIC can simulate PPO cases as well. In a PPO case the energy at the engine burnout, consequently the energy at the beginning of entry, will be lower than that in the nominal flight. The dispersions in other entry conditions will also be increased. For mission mich10a1, if PPO occurs earlier than 50 seconds from liftoff, the entry energy would be too low for the X-33 to reach Michael AAF as the nominal mission calls for. In such a case, a dry lakebed at Ibex Well in Utah near the Nevada border will be targeted as the emergency landing site (Ibex for short). The entry guidance system should ensure that the entry flight trajectory meets all the constraints and TAEM conditions imposed for the nominal mission, regardless of whether it is landing at Michael or Ibex. As a demonstration of the benefits of the on-board trajectory planning capability in these situations, a case of PPO at 60 seconds (still landing at Michael AAF) and another PPO case at 40 seconds (landing at Ibex), both for mission mich10al, will be shown.

The third case is for mission mich10d1. A control surface actuator failure is assumed to have happened at the beginning of entry flight. The consequence is that the maximum bank angle rate and maximum angle of attack rate are both reduced by $25 \%$ to $4 \mathrm{deg} / \mathrm{sec}$. In addition, the vehicle cannot fly as high an $\alpha$-profile as the nominal because of the actuator failure. The angle of attack commands to the control system will be limited up to 5 deg below the nominal values. The landing site is still Michael AAF. The effects of the failure are highly stressful for entry flight in this case: the reduced bank rate increases the time needed to complete the bank reversal during which the bank angle is rate-saturated, thus provides no trajectory control to counter dispersions. The reduction of angle of attack tends to increase the magnitude of the bank angle in order to dissipate the energy, which in turn further lengthens the bank reversal time. Given the short entry flight time of only 2 minutes for mich10d1 mission, more than half of the entry flight duration could be spent on bank reversal maneuver. This would leave little room for the entry guidance system to effectively correct any substantial trajectory dispersions.

These 3 failure cases exemplify the situations where on-board entry trajectory planning capability could make a difference between mission success or failure. The trajectory planning algorithm discussed in this paper proves to be instrumental in achieving mission success in 6DOF MAVERIC simulations. In the case of system failure, the entry guidance system is informed of the failure from the vehicle health monitoring system. The entry trajectory is then generated on-line based on the current entry conditions, the targeted landing site (nominal or alternate), and the reduced control authority of the vehicle in case of actuator failure.

Figure 10 shows the $6 \mathrm{DOF}$ altitude-vs-velocity histories of the entry trajectories for the 
3 cases. Notice that for PPO at 60 seconds, the entry velocity is about $380 \mathrm{~m} / \mathrm{s}$ lower that of the nominal mich10a1 mission as seen in Fig. 4, a reduction of about 14\%. The two PPO mich10al entry trajectories begin with positive flight path angles therefore the altitude keeps increasing before descent starts, a phenomenon not seen in orbital entry. For PPO at 40 seconds, the entry energy (velocity) is even lower hence landing at a closer site (Ibex) is necessary. Figure 11 illustrates the ground tracks of the 3 trajectories and clearly shows to which landing site a trajectory is heading. The histories of heading offset with respect to the HAC versus range-to-HAC are plotted in Fig. 12. The shorter range to Michael AAF of mich10d1 mission is evident. Note that the final ranges are all close to the required value of $55.56 \mathrm{~km}$, and the largest final heading offset is only 1 deg among all the cases. Table 2 summarizes the TAEM conditions errors for the 3 cases in $6 \mathrm{DOF}$ simulations. Compared to the required values as stated earlier, the TAEM conditions are met remarkably well, given the severity of the failures.

Finally Fig. 13 depicts the variations of bank angle for the 3 entry trajectories where the time is counted from launch (so the abscissa starts at about 310 seconds). Particularly revealing is the bank history for mich10d1 mission with actuator failure. The total entry flight time is about 120 seconds, but half of it is needed just for the bank reversal at reduced maximum bank rate. Coupled with reduced angle of attack, this characteristics makes it significantly more difficult for the entry guidance system to achieve accurately the required TAEM conditions. In fact it is impossible to fly the nominally designed mich10d1 reference trajectory in this case. Without an on-board trajectory planner that designs a reference entry trajectory specifically tailored to the current situation, it would be highly unlikely for any entry guidance system to successfully guide the vehicle for a safe landing should this failure happen to mich10d1 mission.

Table 2: TAEM condition precision (6DOF) for cases with system failures

\begin{tabular}{ccccc}
\hline Case & $\Delta \psi_{f}(\mathrm{deg})$ & $\Delta s_{f}(\mathrm{~km})$ & $\Delta r_{f}(\mathrm{~km})$ & $\sigma_{f}(\mathrm{deg})$ \\
\hline \hline & & & & \\
mich10a1 PPO@60 sec & 0.55 & 1.417 & -0.11 & 14.1 \\
mich10a1 PPO@40 sec & 0.99 & -0.001 & 0.399 & -25.3 \\
mich10d1 w/ actuator failure & 1.03 & -2.137 & 0.595 & 34.1 \\
\hline \hline
\end{tabular}

\section{Conclusions}

A method for fast planning of sub-orbital entry trajectories is developed. The algorithm generates a complete feasible three-degree-of-freedom sub-orborbital entry trajectory within a fraction of one second on a desktop computer, making it suitable for on-board applications. Extensive high-fidelity simulations using a reusable launch vehicle model and missions have validated the effectiveness and reliability of the algorithm. This algorithm complements a recently developed on-board entry trajectory planning algorithm for orbital entry missions. With the current development, the capability now exists for full-envelope on-board entry trajectory planning. Such a capability could make future space transportation systems safer, more adaptive and cost effective. 


\section{Acknowledgments}

This research has been supported in part by NASA Contract NAS8-01105 and NASA Grant NAG8-1637. 


\section{References}

1 Harpold, J. C., and Graves, C. A., "Shuttle Entry Guidarice," The Journal of the Astronautical Sciences, Vol. XXXVII, No. 3, 1979, pp. 239-268.

${ }^{2}$ Gamble, J. D., Cerimele, C. J., Moore, T. E., and Higgins, J., "Atmospheric Guidance Concepts for an Aeroassisted Experiment," The Journal of the Astronautical Sciences, Vol.36, No. 1-2, 1988, pp. 45-71.

3 Fuhry, D. P., "Adaptive Atmospheric Reentry Guidance for the Kistle K-1 Orbital Vehicle," Proceedings of AIAA Guidance, Navigation, and Control Conference, Vol. 2, pp. 1275-1288, 1999, AIAA, Reston, VA.

4 Zimmerman, C., Dukeman, G., and Hanson, J., "An Automated Method to Compute Orbital Entry Re-Entry Trajectories with Heating Constraints", AIAA paper 2002-4454, Guidance, Navigation, and Control Conference, Monterey, CA, August 5-8, 2002

5 Mease, K. D., Chen, D. T., Teufel, P., and Schoenenberger, H., "Reduced-Order Entry Trajectory Planning for Acceleration Guidance", Journal of Guidance, Control, and Dynamics, Vol. 25, No. 2, 2002, pp. 257-266.

6 Roenneke, A. J., "Adaptive On-board Guidance for Entry Vehicle," AIAA Paper 20014048, Guidance, Navigation, and Control Conference, Montreal, Canada, August 6-9, 2001.

7 Shen, Z., and Lu, P., "On-Board Generation of Three-Dimensional Constrained Entry Trajectories", to appear in Journal of Guidance, Control, and Dynamics, Vol. 26, No. $1,2003$.

8 Barton, G. H., Grubler, A. C., and Dyckman, T. R., "New Methodologies for Onboard Generation of TAEM Trajectories for Autonomous RLVs" , Core Technologies for Space Systems Conference, November19-21, 2002, Colorado Springs, CO.

9 Vinh,-N.-X., Busemann, A., and Culp, R: D., Hypersonic and Planetary Entry Flight Mechanics, The University of Michigan Press, Ann Arbor, MI, 1980, pp. 26-27

10 Hanson, J., "Advanced Guidance and Control Project for Reusable Launch Vehicles," AIAA Paper 2000-3957, Guidance, Navigation, and Control Conference, Denver, CO, August 14-17, 2000.

11 Lu, P., "Regulation about Time-Varying Trajectories: Precision Entry Guidance Illustrated," Journal of Guidance, Control, and Dynamics, Vol. 22, No. 6, 1999, pp. 784-790.

12 Justus, C. G. and Johnson, D. L., The NASA/MSFC Global Reference Atmospheric Model: 1999 Version (GRAM-99), NASA TM-209630, May, 1999

13 Dukeman, G. A., "Profile-Following Entry Guidance Using Linear Quadratic Regulator Theory", AIAA Paper 2002-4457, Guidance, Navigation, and Control Conference, Monterey, CA, August 5-8, 2002. 


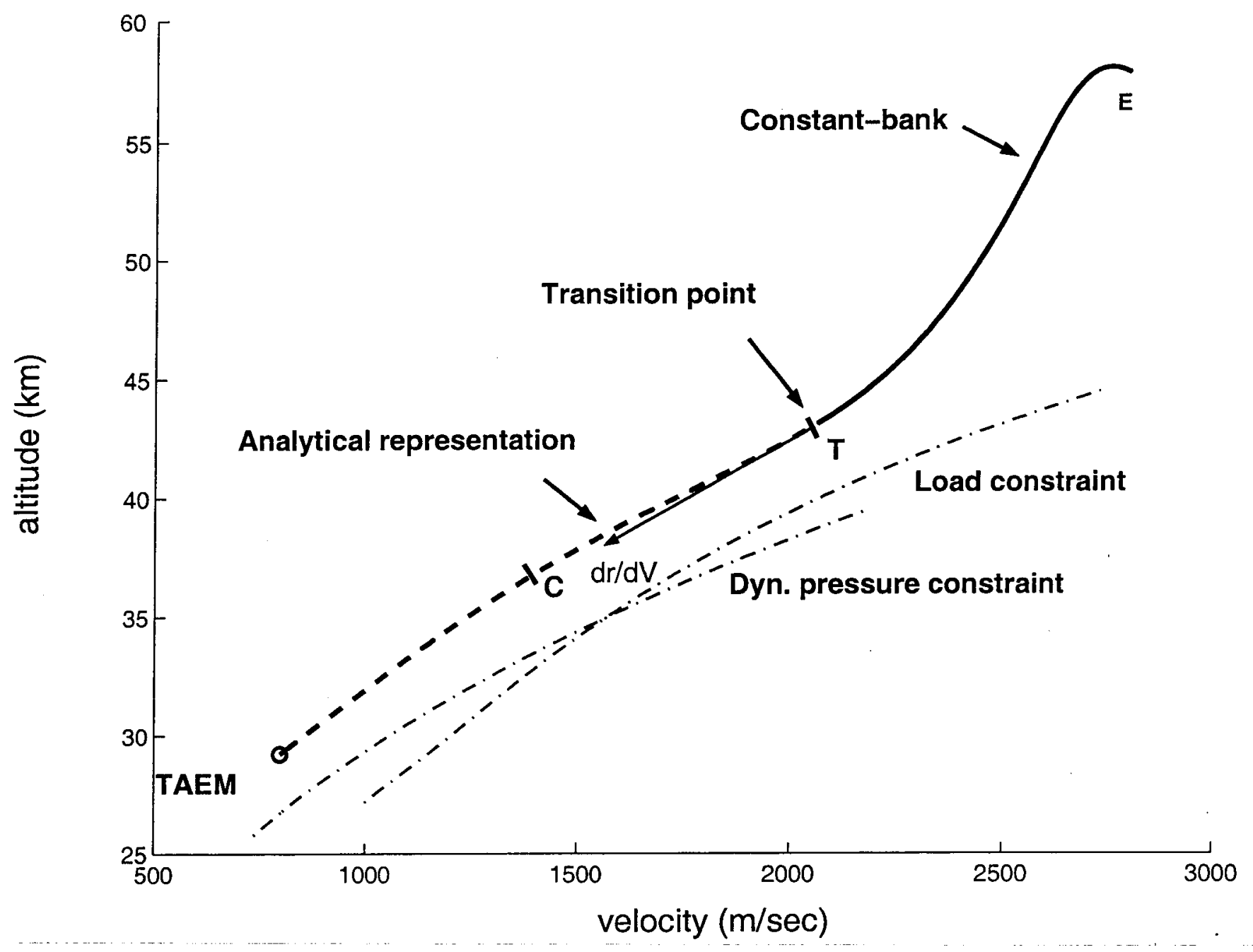

Fig. 1: Longitudinal profile 


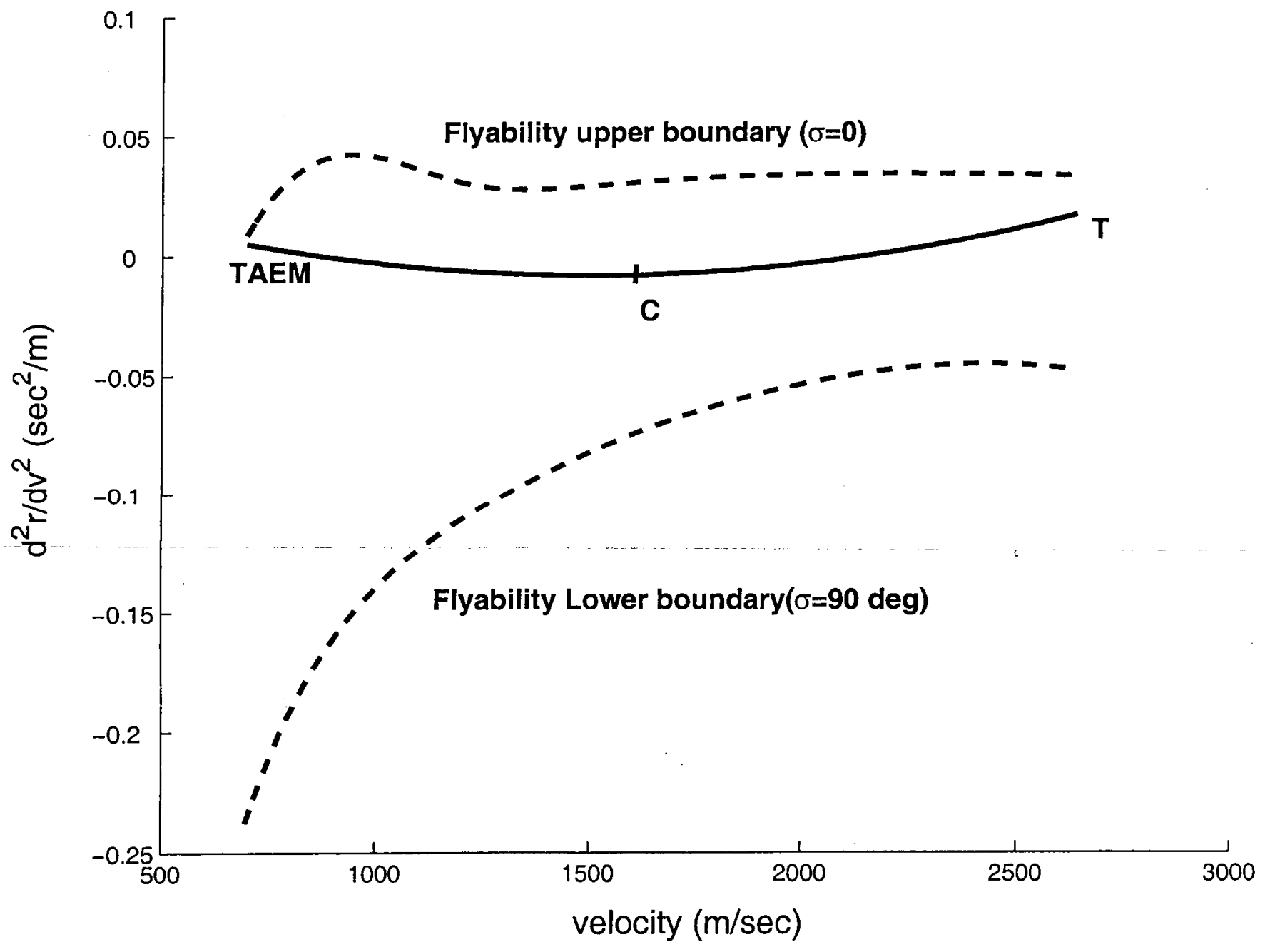


Fig. 2: Flyability corridor

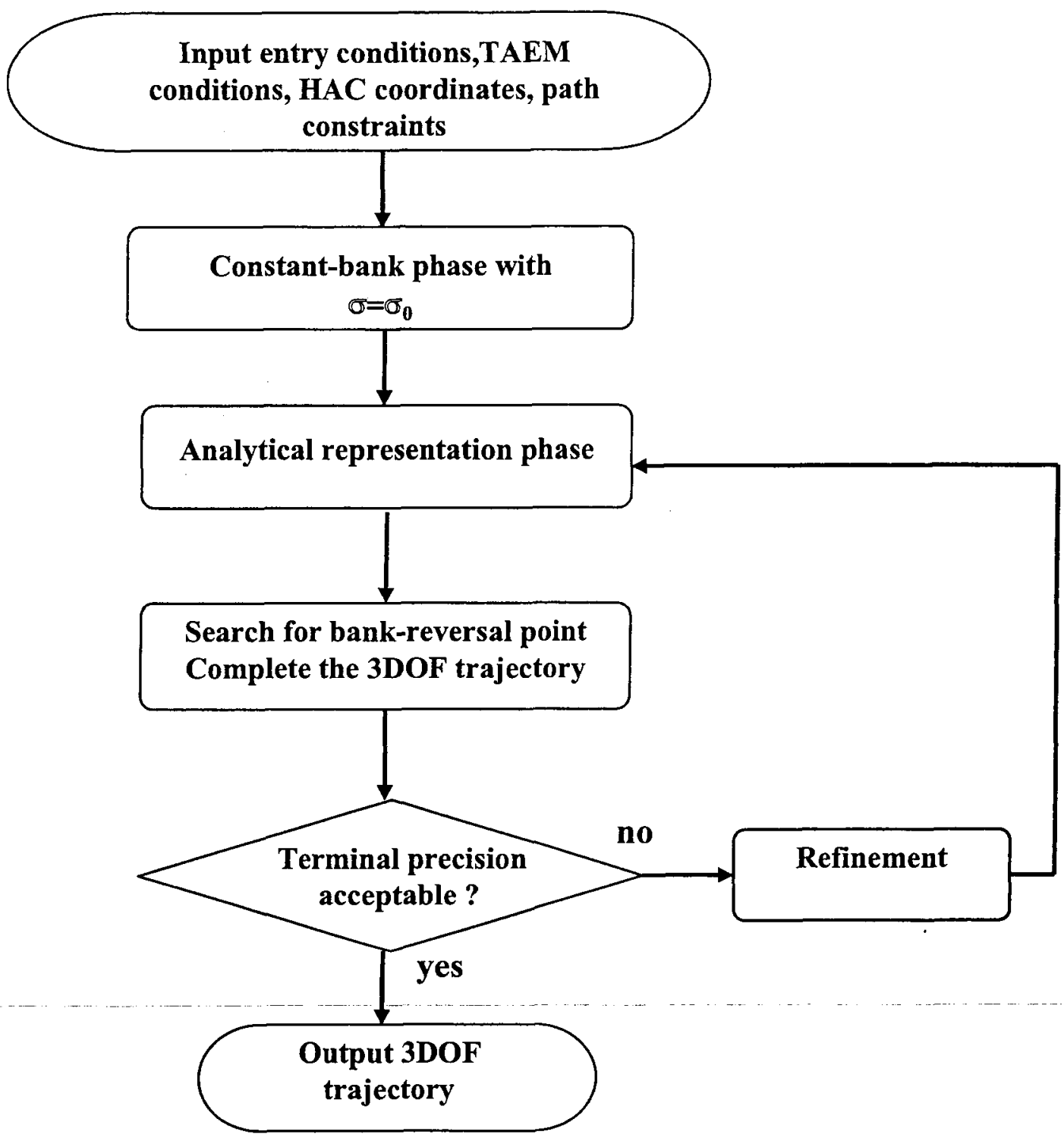

Fig. 3: Algorithm flowchart 


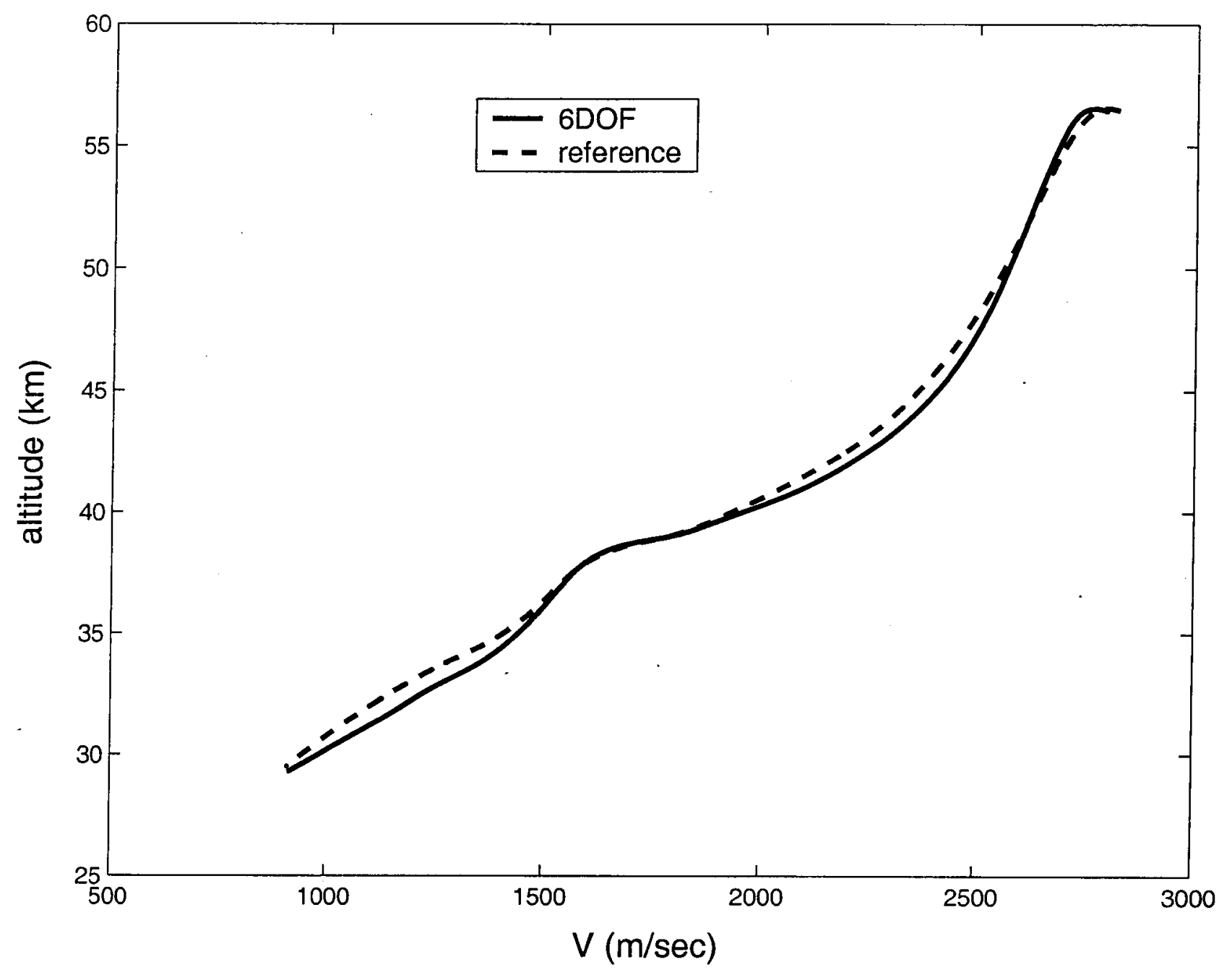

Fig. 4: Entry trajectory for mission mich10a1 

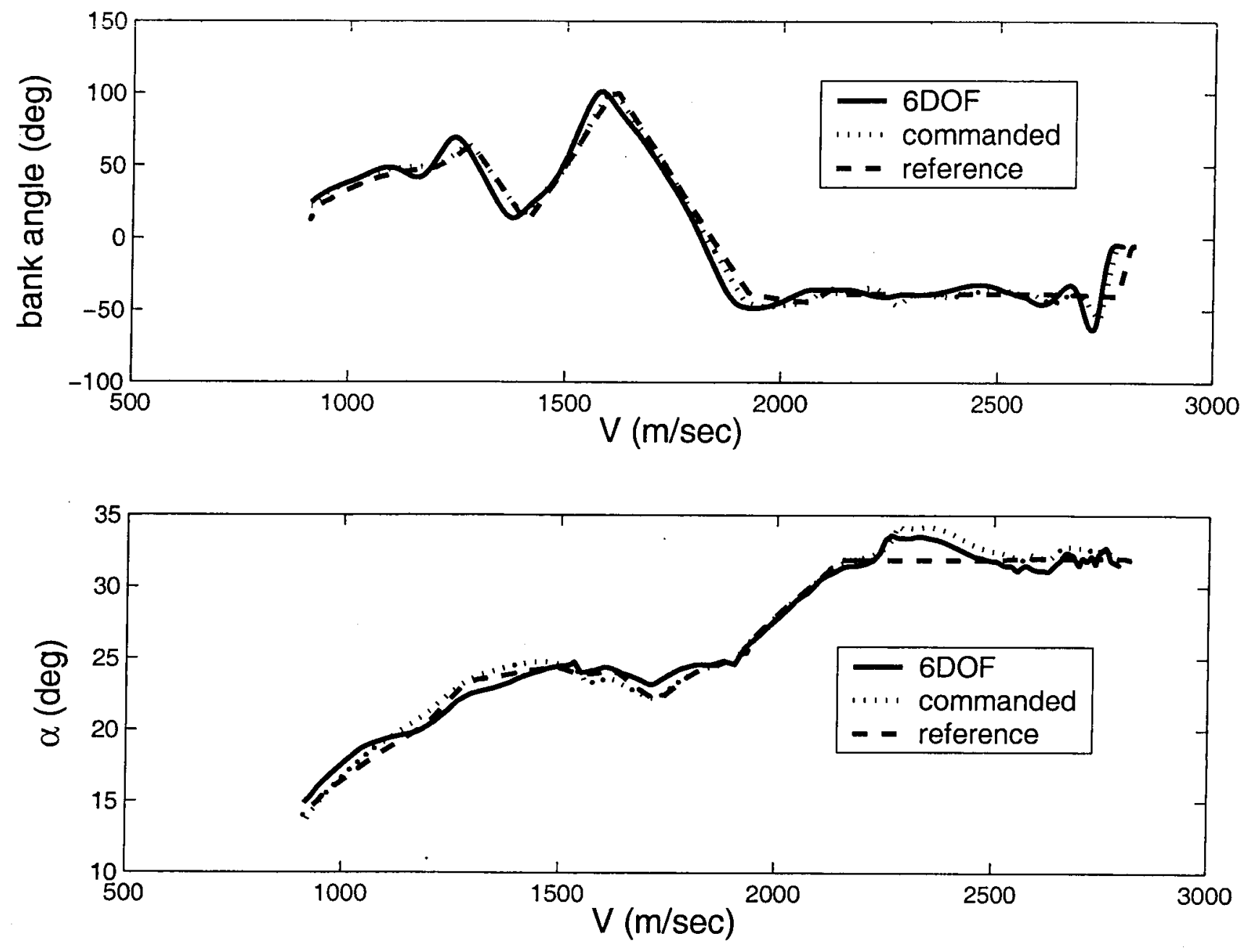

Fig. 5: Bank angle and $\alpha$ histories for mich10a1 


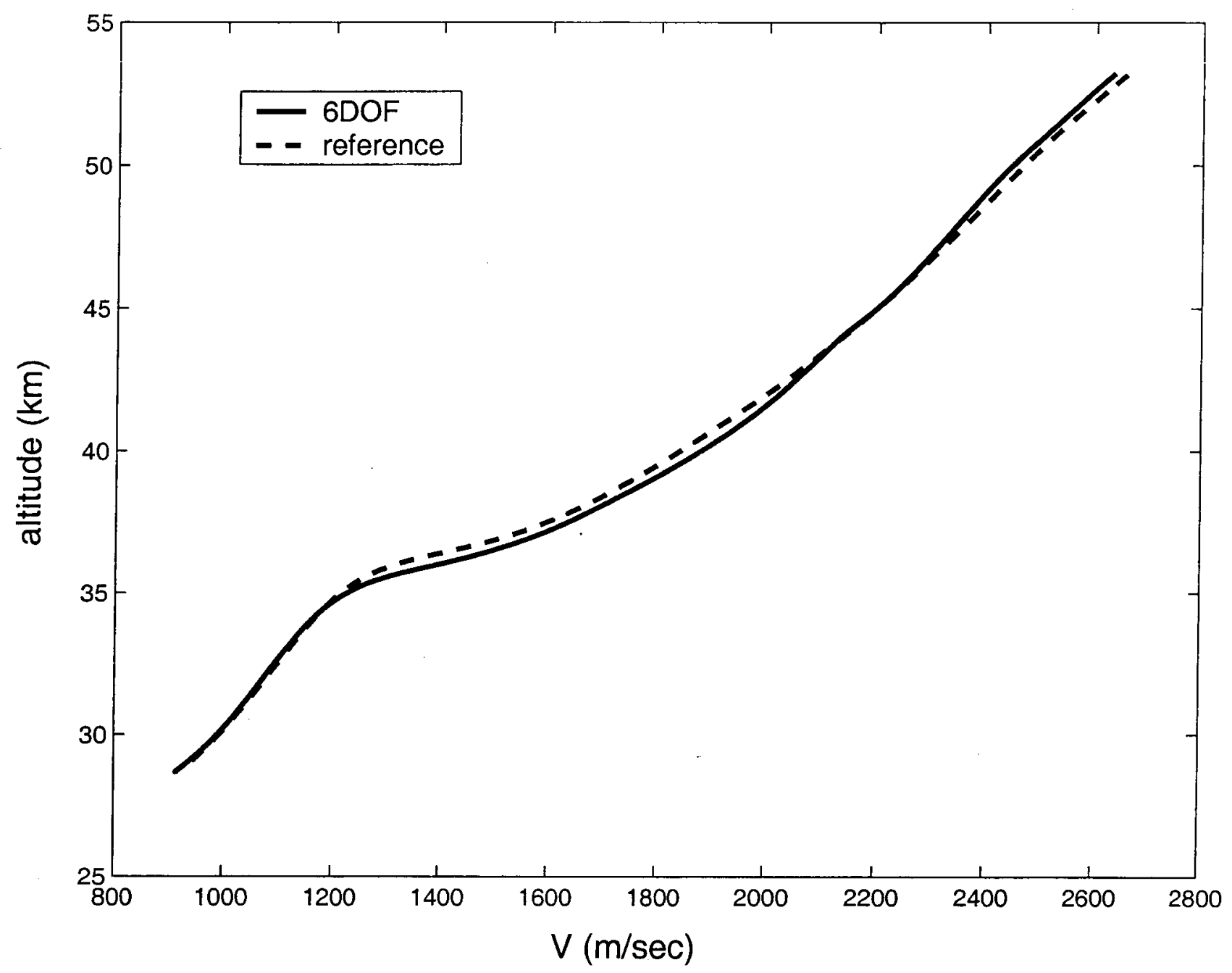

Fig. 6: Entry trajectory for mission mich10d1 

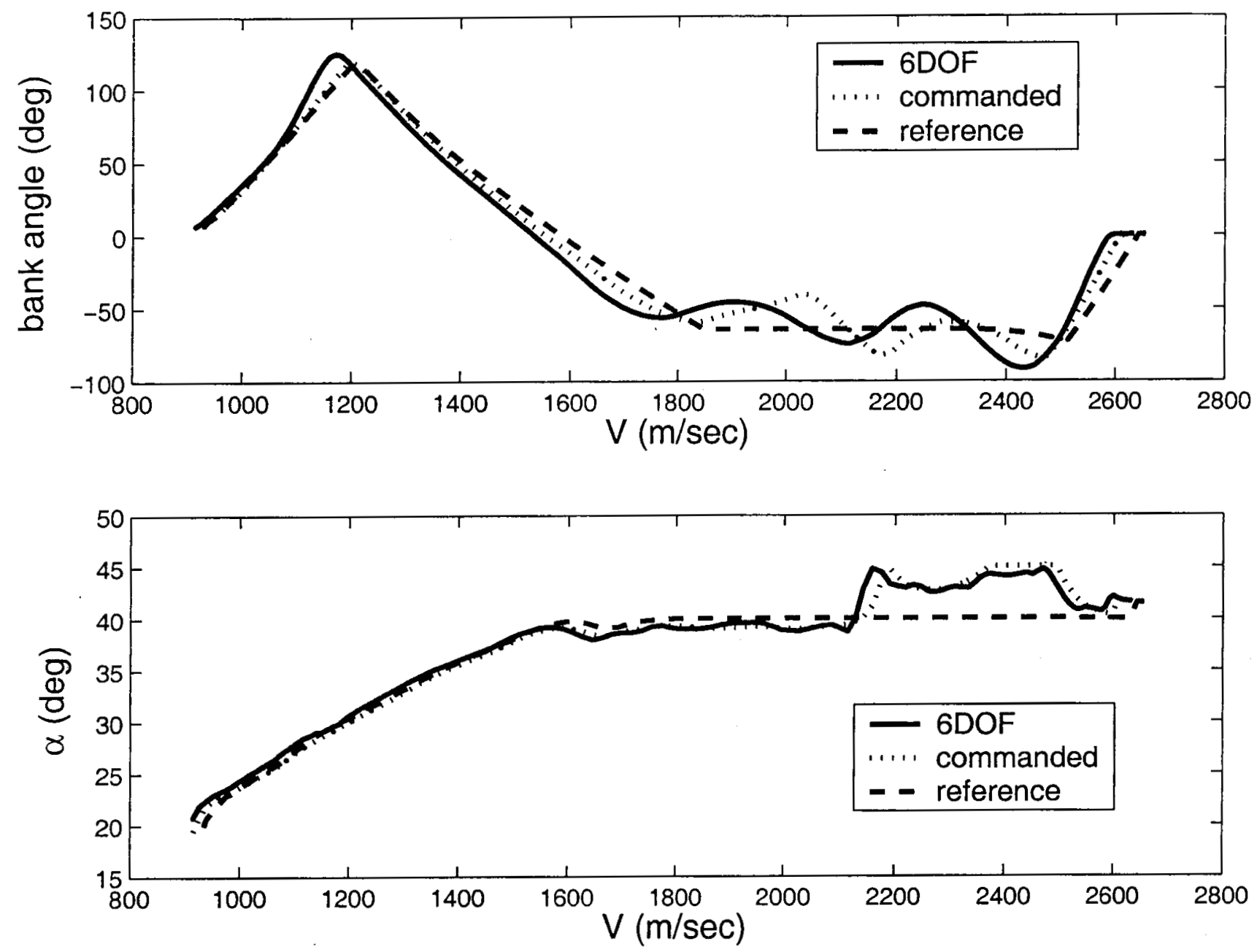

Fig. 7: Bank angle and $\alpha$ histories for mich10d1 


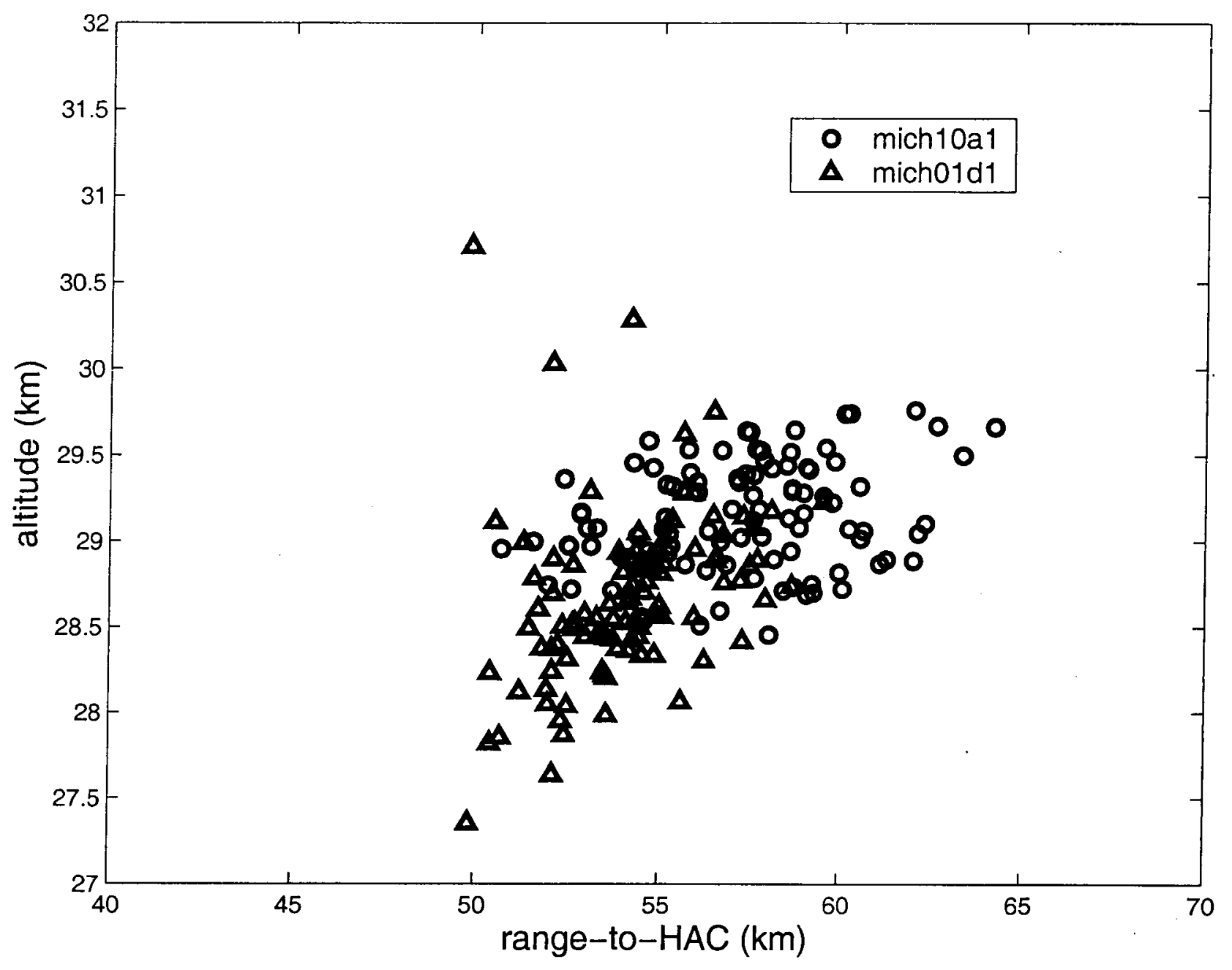

Fig. 8: TAEM altitudes and ranges for 100 dispersed mich10a1 and 100 mich10d1 trajectories 


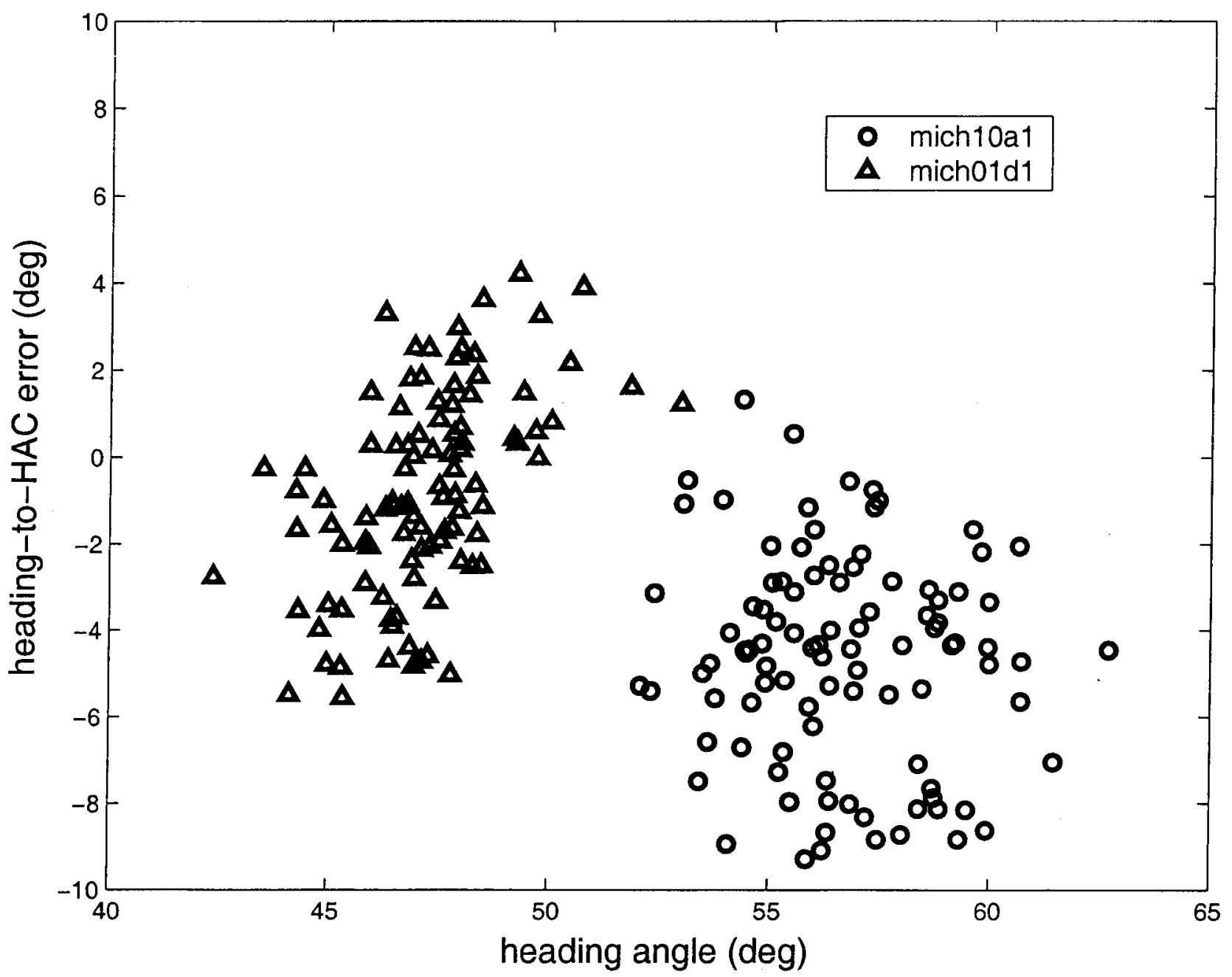

Fig. 9: TAEM heading angles and heading-to-HAC errorfor 100 dispersed mich10a1 and 100 mich $10 \mathrm{~d} 1$ trajectories 


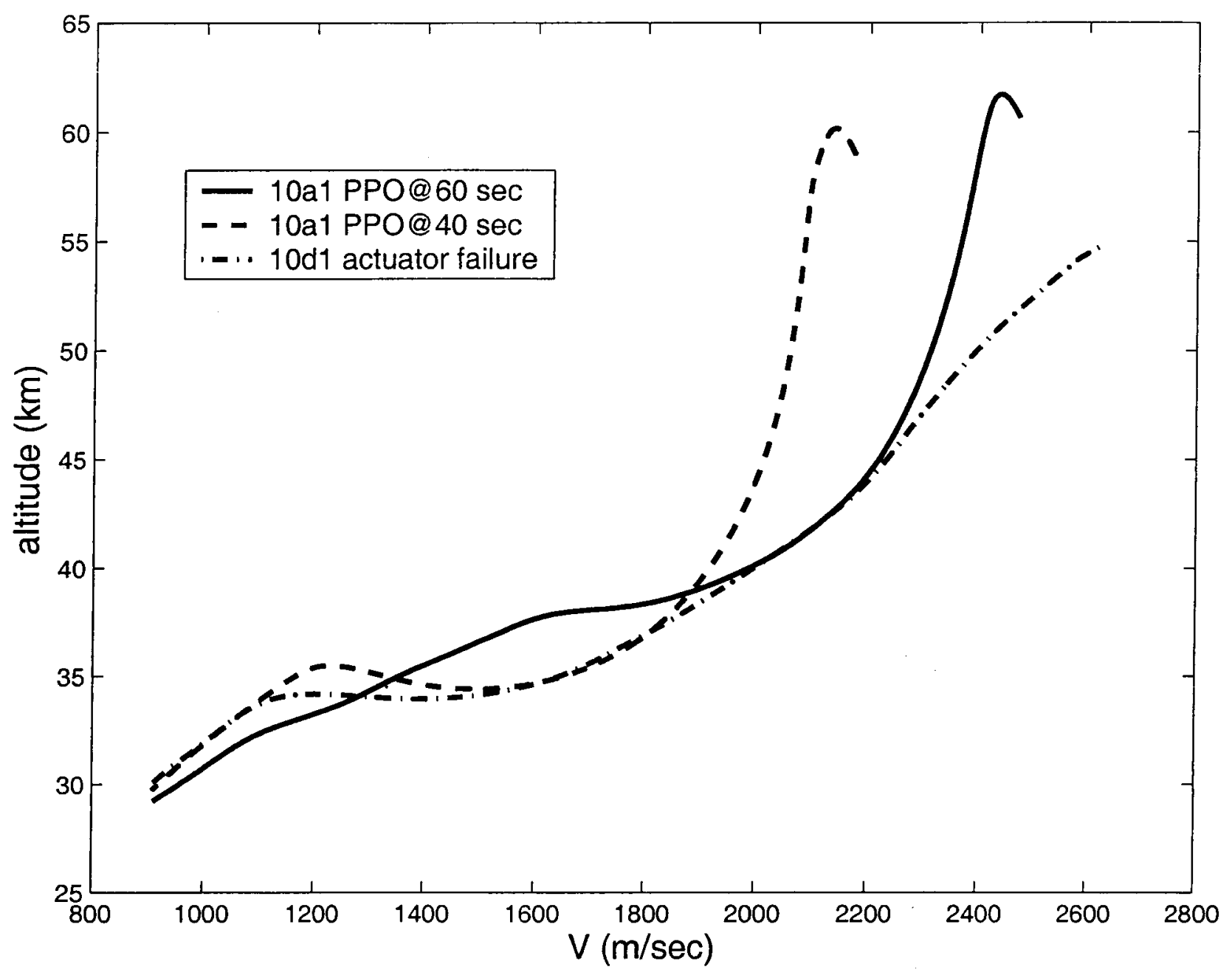

Fig. 10: Entry trajectories of 3 cases with system failures 


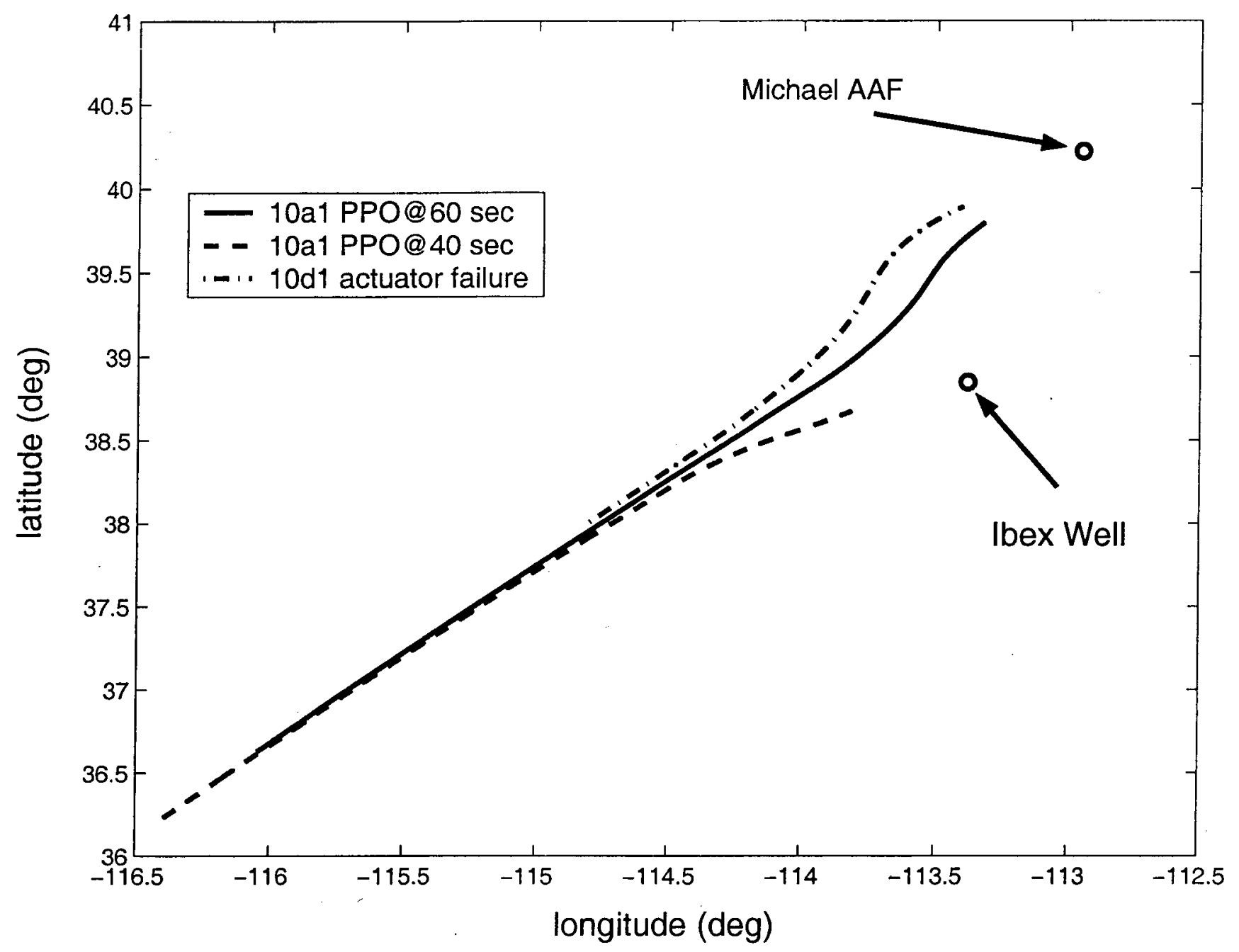

Fig. 11: Ground tracks of the entry trajectories with system failures 


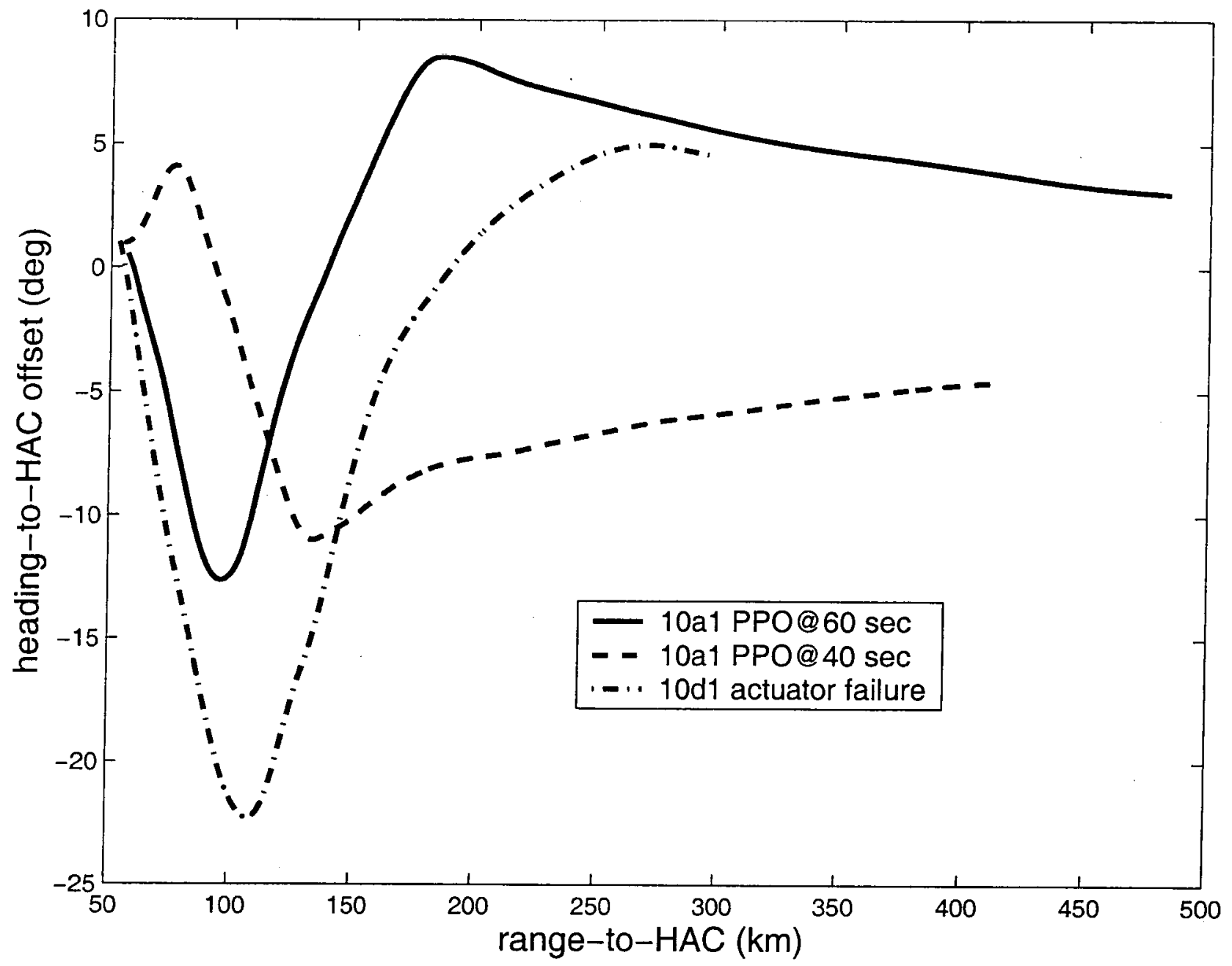

Fig. 12: Heading angle offset with respect to HAC of the entry trajectories with system failures 


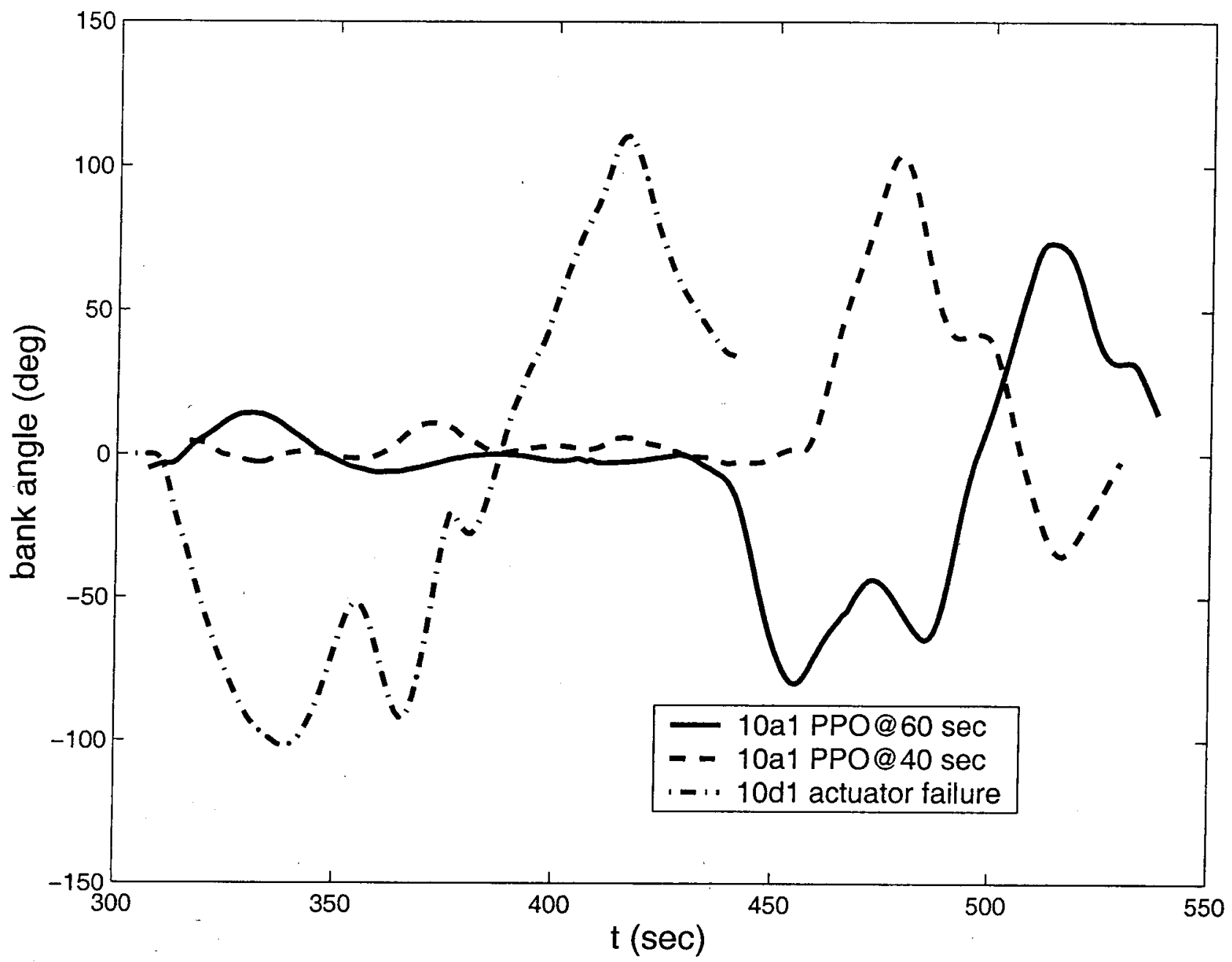

Fig. 13: Bank angles along the entry trajectories with system failures 